a machine-independent binary-file standard for recording matrix arrays and other types of scientific data, which can be quite extensive. This format permits flexibility; for example, subarrays corresponding to part of an image can be accessed for read/write purposes. Data are stored as 1- or 3-byte integers, or as floatingpoint numbers, in binary-format continuous-byte file (not subdivided into blocks).

We see both TIFF and HDF as suitable formats for microscopy. In terms of immediate use, TIFF has the largest amount of support; it is used by all IBM-PC and Macintosh page-scanner programs, by page-layout applications such as Ventura Publisher, QuarkXPress and Aldus PageMaker, and by image-editing applications such as Image, Digital Darkroom, Photoshop, ImageStudio, ImageEdit and Snapshot. Because these applications typically read several formats, they can be used to translate TIFF files into other forms. TIFF is flexible and evolving; it currently allows multiple images per file, several types of data compression, and various types of color image. Because the image data is organized into 'strips', an application program can call into memory only those parts of an image which require processing. TIFF documentarian is available over the Internet network form several FTP sources, including zippy. nimh nih gov (see the /pub directory).

Microscopists should remain aware of the HDF standard, which might grow in popularity within the scientific community. NCSA's program Import2HDF can read several 'foreign' formats including TIFF, FITS, PICT, GIF and ASCII Text Files. HDF documentation, source code and Macintosh applications are available free of charge (via anonymous FTP) over Internet (ftp ncsa uiuc edu)

MSA Standards Committee Report, MSA Bulletin, vol 23, No.2, 1993

Reprinted from Proccedings, 51st MSA Annual Meeting, with the permission of the Microscopy Society of America. Authors include members of the MSA Standards Committee. R.F Egerton (Univ of Aiberta), D.S. Bright (NIST), S.D. Davilla (4pi Systems), P. Ingram (Duke Univ.). EJ. Kirkland ((Cornell Univ.), M. Kundmann ((Gatan, Inc.). C.E. Lyman ((Lehigh Univ). P Rez (Arizona State Univ.), E Steele (NSST) and N.J. Zaluzec (Argonne Nat'l Lab.)

\section{Projector Slide Plates}

A. Kent Christensen, Univ. of Michigan Medical School

The preparation of electron micrographs for $2^{11} \times 2^{\prime \prime}$ slide presentations is usually done by photographing EM prints with a $35 \mathrm{~mm}$ camera. However, for some of our better electron micrographs, many of us have enjoyed printing the negatives directly on $2^{\prime \prime} \times 2^{\prime \prime}$ Kodak Projector Slide Plates (\#s 1406875 \& 140 6867 ), which can yield projected images of particularly pleasing tonal quality.

A previous issue of Microscopy Today (issue 94-2, March 1994) contained a brief article my me describing a method for making 2" $\times 2$ " EM slides with Projector Slide Plates. Unfortunately, it now appears that Kodak has raised the price of these plates to a level that is well beyond what most of us can afford. My first inkling of this came after the article was in the hands of the editor. I telephoned Kodak and confirmed that the price was now about $\$ 560$ per box of 36 plates, roughly 10 times the former price. However, it still seemed possible that this had been an oversight on the part of Kodak, and that it could be rectified. After another telephone call and a letter, I was finally able to speak on the phone with a Kodak executive, who explained the situation. He said they have had great difficulty getting the emulsion gel components for their various glass plate products and that there was a great deal of waste in manufacturing. As a result, the production of their glass plate products had been operating at a loss for a considerable time. In this era of extremely tight budgets, the Kodak marketing people decided that they had to set realistic prices for their glass plate products that reflected the real cost of making them, plus a reasonable profit. By the time all of this had been clarified, my article was already in press in Microscopy Today.

We regret that this fine product is now out of the reach of most electron microscopists. It looks like we will all be using $35 \mathrm{~mm}$ copies for our future EM projection slides. Maybe we can print EM negatives on sheet film. Or perhaps the new wave of digitized EM images on computers will allow eventually to make $2^{\prime \prime} \times 2^{\prime \prime}$ slides that have whatever tonal quality we desire.

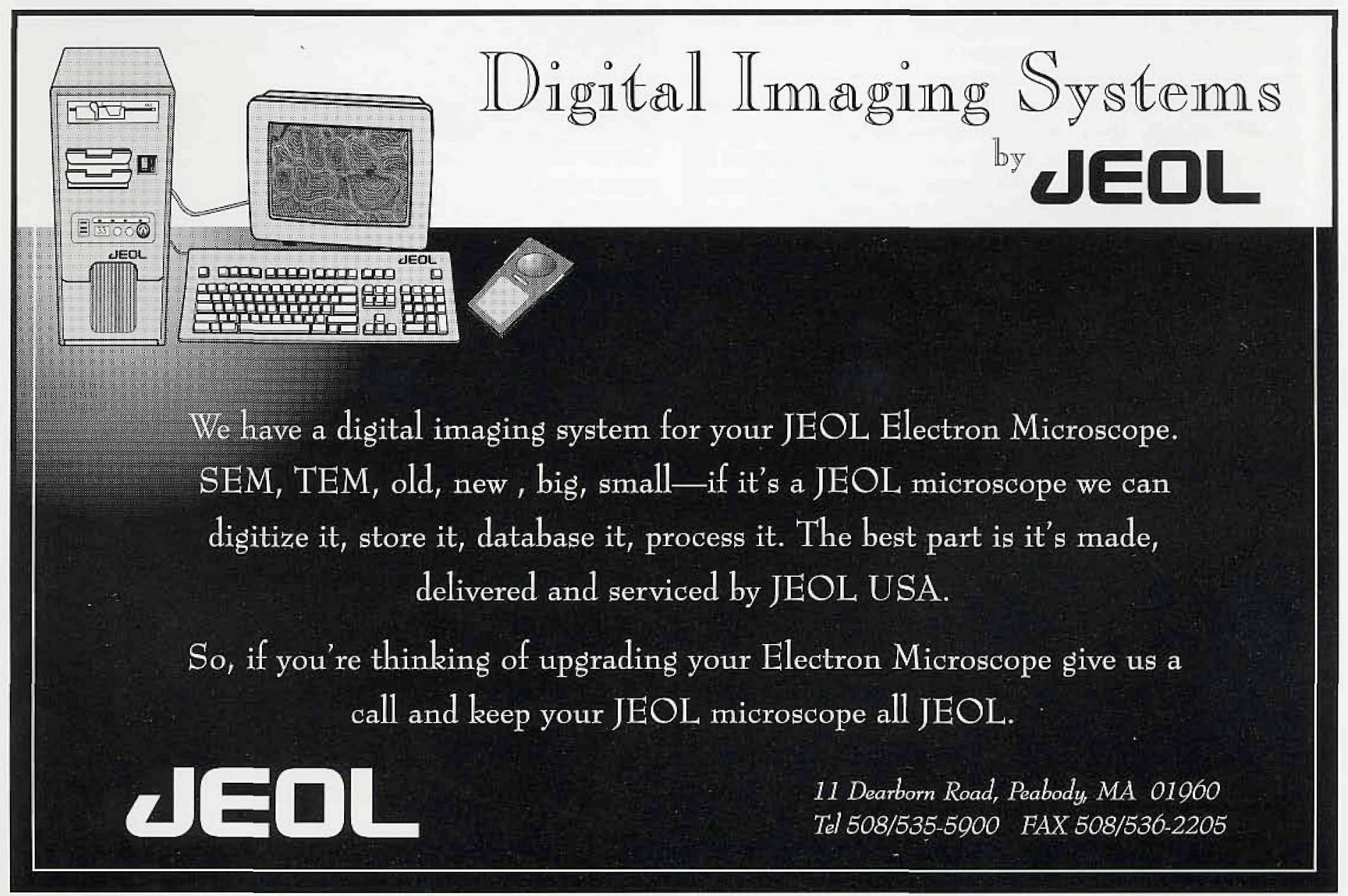

